\title{
Après la mort de Georges Olivet
}

\author{
délégué du Comité international de la Croix-Rouge ${ }^{1}$
}

Georges Olivet est mort au service de la Croix-Rouge, en décembre I96I, près d'Elisabethville, au Katanga. Le dernier message reçu de lui date du I3 décembre, et il y annonçait qu'il s'était présenté à plusieurs reprises sans succès, au quartier général des Nations Unies, les soldats de garde ayant refusé de le laisser passer. Il voulait y négocier une trêve pour l'évacuation des civils se trouvant dans les zones de combats.

Le I4 décembre, des bruits commencent à circuler selon lesquels M. Olivet aurait disparu; le CICR demande aussitôt que des recherches soient entreprises par les Forces des Nations Unies au Katanga. Toujours sans nouvelles le jour suivant, le CICR demande à son délégué à Léopoldville de se rendre immédiatement à Elisabethville pour participer aux investigations.

Les jours suivants, des télégrammes s'échangent entre Elisabethville, New-York et Genève, et il se confirme que c'est bien le I3 que M. Olivet a été vu pour la dernière fois, se dirigeant dans une ambulance vers le quartier général des Nations Unies, accompagné de Mme Nicole Vroonen et de M. Styts Smeding, tous deux volontaires de la Croix-Rouge katangaise. Les délégués du CICR, MM. Senn et de Preux, de même que M. Melcher, correspondant, demeurent constamment en relation avec les personnalités des Nations Unies de qui dépendent l'enquête. N'ayant pas réussi à réunir encore des

${ }^{1}$ Hors-texte 


\section{Georges Olivet,}

mort au service de la Croix-Rouge (

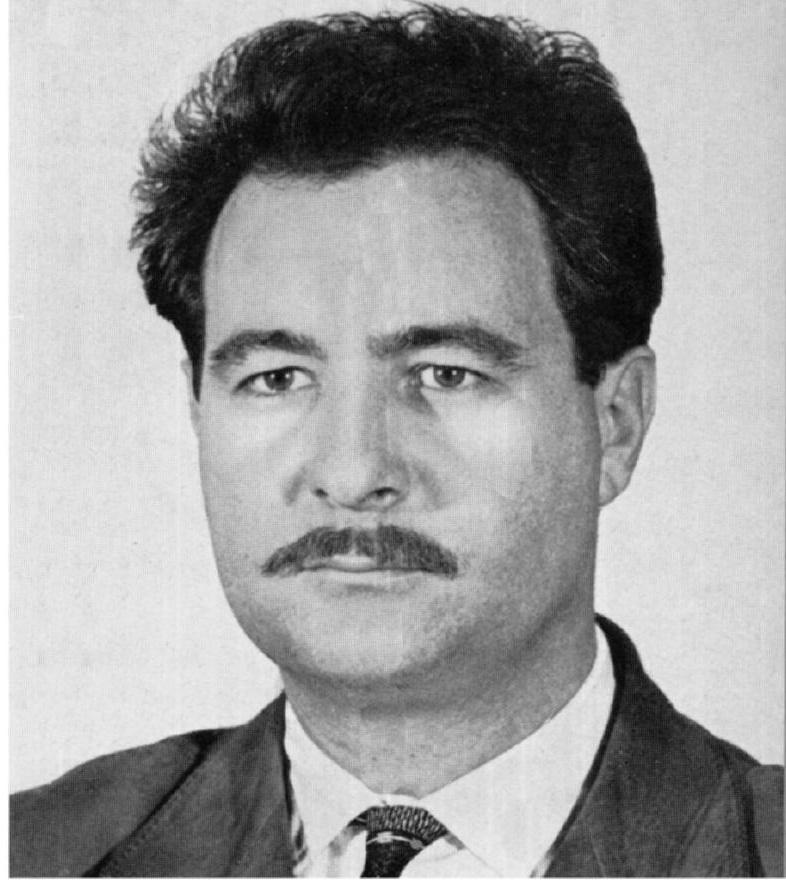

Le voici distribuant des secours, à Luena, au Congo. en ro6o.

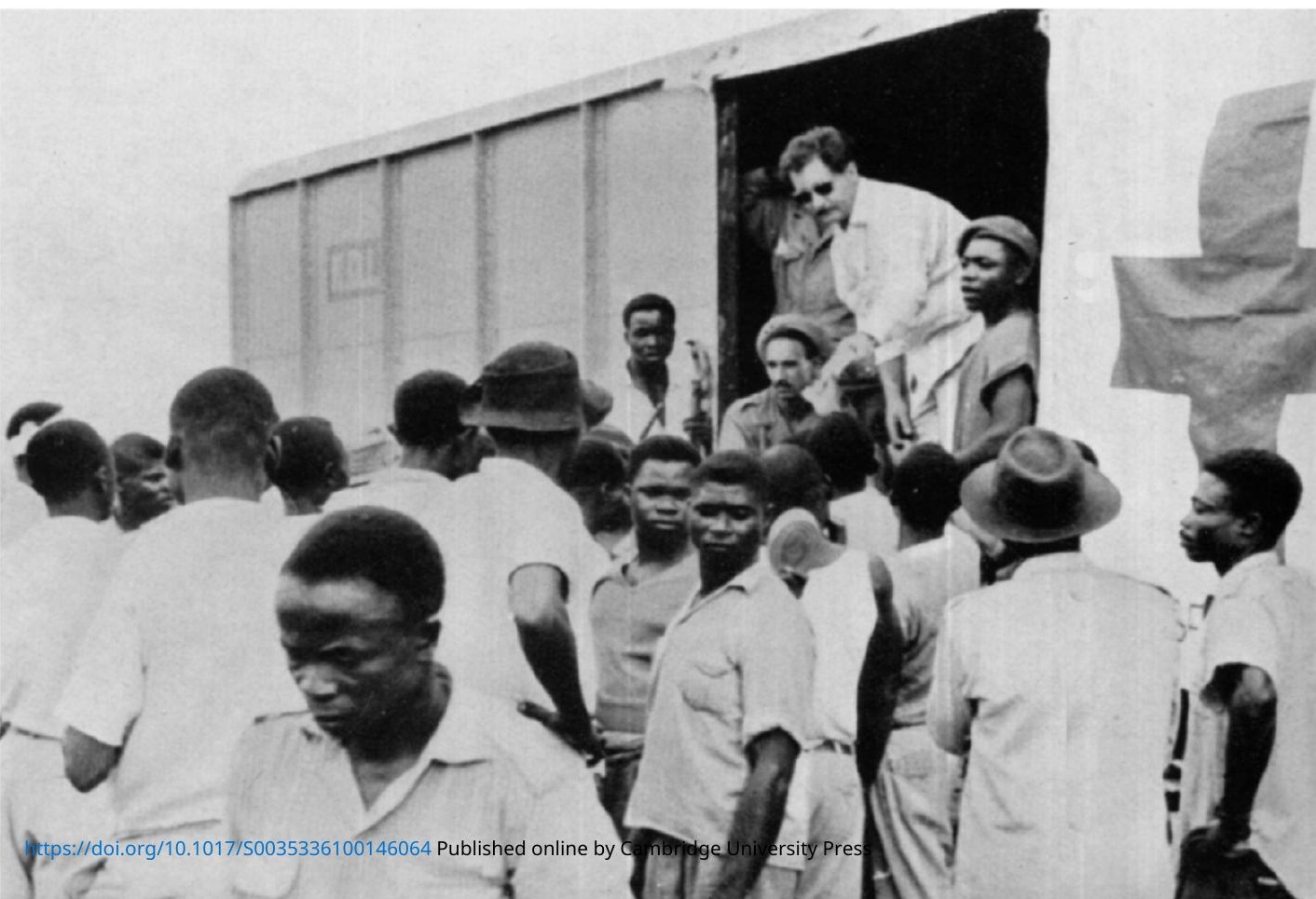


indications valables, ils prévoient des patrouilles militaires aux fins de rechercher les traces des trois disparus.

Inquiet cependant par le silence persistant, le Comité international décide, le 22 décembre, d'envoyer de Genève à Elisabethville une personnalité indépendante avec la tâche d'enquêter sur le sort de M. Olivet; il en avise le secrétaire général des Nations Unies et celui-ci répond en assurant le CICR « que l'ONU n'épargnera aucun effort pour retrouver M. Olivet, dont nous estimons, dit-il, très hautement l'œuvre accomplie au Katanga ».

Toutefois, le lendemain, un télégramme du correspondant du CICR à Elisabethville parvient à Genève, qui est conçu en ces termes:

"Corps Olivet et madame Vroonen de nationalité belge et Smeding de nationalité hollandaise trouvés ce matin. Corps se trouvaient ensevelis à 20 mètres environ de l'ambulance. Ambulance a été touchée par mitraillade et tir bazooka ou autre. Ambulance se trouvait le $\mathbf{I}_{3}$ décembre en pleine zone de combat, enquête faite par procureur d'Etat en cours. Vous tiendrai au courant du résultat dès que possible. Avons ramené corps madame Vroonen à l'hôpital Reine Elisabeth. Deux autres corps seront déterrés cet aprèsmidi ».

Ainsi, le drame était consommé et c'est avec la plus profonde affliction que le Comité international apprit cette nouvelle, qui devait être confirmée par la suite, et qu'il eut la douloureuse mission d'apprendre à la famille de M. Olivet. Mais les circonstances mêmes de ce terrible événement n'étaient pas élucidées et, le 24 décembre, le CICR, n'ayant pas obtenu entre-temps des renseignements suffisants, annonçait à $M$. Thant et à $M$. Tschombé le départ de l'un de ses vice-présidents, M. F. Siordet, pour Elisabethville. Nous reviendrons sur les résultats de cette mission.

Enfin, le 25 décembre, le CICR recevait un nouveau télégramme de son correspondant à Elisabethville, tragique dans sa brièveté, et par lequel celui-ci précisait que le matin même, Georges Olivet et ses compagnons avaient été inhumés dans l'enclos de l'hôpital Reine Elisabeth en présence des délégués du CICR, du représentant du Gouvernement katangais, du corps consulaire, des représentants de la Croix-Rouge katangaise et du corps médical. 
Dès que fut confirmée, à Genève, la terrible nouvelle du décès de son représentant au Katanga, le Comité international de la Croix-Rouge publia, le 24 décembre I96I, le communiqué suivant :

Le Comité international de la Croix-Rouge, à Genève, a reçu de sa délégation au Congo confirmation du décès de son représentant, M. Georges Olivet, mortellement frappé, avec deux membres de la Croix-Rouge du Katanga, alors qu'il se portait au secours de victimes des combats à Elisabethville.

Le CICR tient à rendre publiquement hommage à ce fidèle serviteur de la Croix-Rouge, qui n'a cessé, depuis le début des hostilités au Congo, de s'exposer, avec un courage exemplaire, pour soulager les souffrances et sawver nombre d'êtres humains. Il a payé de sa vie son dévouement à l'idéal de la Croix-Rouge.

Un des vice-présidents du CICR, M. Frédéric Siordet, va se rendre au Congo pour assister à l'enquête ouverte par les autorités.

Puis M. Boissier, président de l'institution, rendit à M. Olivet, dans la presse et à la radio, l'hommage que voici :

On se souvient de l'affirmation célèbre; "Je ne crois qu'aux causes pour lesquelles on se fait tuer". Pour la Croix-Rouge ou, plutôt, pour ceux qu'elle secourt, on se fait twer et le Comité international de la Croix-Rouge compte un martyr de plus.

Depuis dix-huit mois, les délégués du CICR ont parcouru le Congo, en auto sur les chemins dangereux à la merci d'une embuscade, dans les avions qui atterrissent tant bien que mal sur des pistes souvent bloquées. Partout, ils ont cherché à délivrer des prisonniers, à échanger des otages, à protéger des populations menacées du massacre et de la tamine.

Hier encore, Georges Olivet se dépensait sans compter avec un amour, une foi et un courage que tous, Blancs et Noirs, admiraient. Car cet être était prédestiné à accomplir une telle mission. Parlant plusieurs langues indigènes qu'il avait apprises dans sa pratique de commerçant, il savait entrer en contact direct avec les populations locales, leur parler, les comprendre et, surtout, s'en faire aimer.

On lui faisait confiance et on l'appelait au secours. Pour lui, une seule chose comptait: se dévouer entièrement à une cause à laquelle il avait consacré sa vie. 
C'est ainsi qu'à Elisabethville, au milieu de combats, il chercha, à plusieurs reprises, à franchir les lignes ennemies pour sawver les victimes d'un feu meurtrier. Il n'y réussit pas. Il essaya encore et ce fut la fin. La fin d'une existence terrestre, mais le début d'un exemple, d'une haute leçon.

Lorsque débutèrent, en I960, les événements graves qui allaient déchirer le Congo, Georges Olivet, dans un élan spontané, s'était mis à la disposition du CICR. Tous ceux qui devinrent alors ses compagnons, à la délégation du CICR à Léopoldville, rendirent témoignage de son courage et de sa générosité. Serviable, toujours prêt à aider, aimant le Congo qu'il connaissait bien, puisqu'il y était établi depuis plus de six ans déjà, il fit toujours montre, dans les missions qu'il entreprit avec ses collègues, du meilleur esprit de camaraderie et, en toutes circonstances, il témoigna de son dévouement à l'idéal qu'il a servi dans un mouvement intérieur profond, et pour lequel il est mort.

Le 29 décembre I96r, eut lieu, dans l'un des temples de Genève, une émouvante cérémonie à sa mémoire, cérémonie à laquelle assistaient la famille du défunt, les représentants des autorités cantonales et municipales, de la Ligue, ainsi que de nombreuses institutions internationales et des représentants diplomatiques de plusieurs pays. Un grand drapeau à croix rouge était déployé devant le chœur et le président, de même que de nombreux membres et collaborateurs du CICR, avaient pris place dans l'église.

Au nom du Comité international, M. Léopold Boissier évoqua dans les termes qu'on va lire, celui qui a payé de sa vie son dévouement à la cause de la Croix-Rouge :

Au début de juillet x96o, le Congo belge, devenu indépendant, est en pleine effervescence. Le désordre règne et l'unique représentant du Comité international de la Croix-Rouge, M. Ammann, à peine arrivé, se trouve devant une tâche surhumaine.

Par bonheur, un jeune commerçant genevois se présente à lui. Il offre ses services à titre bénévole. Ses services, c'est d'abord sa machine à écrire, son automobile, sa bonne volonté et tout le temps qu'on voudra. C'est aussi sa connaissance du pays, de ses idiomes, de ses coutumes, 
de ses ethnies et de ses chefs. C'est encore son intérêt pour ces indigènes qu'il comprend et qu'il aime, et qui lui rendent au centuple sa compréhension et son affection.

Mais, par-dessus tout, ce qui est offert par Georges Olivet c'est son être tout entier, c'est sa vie même, dont il fera le don suprême à la cassure du chemin où sa destinée chrétienne le conduit.

Pour certains hommes, la rencontre de la Croix-Rouge est un moment décisif de leur existence. Une vaste entreprise les invite à employer le meilleur d'eux-mêmes à une ceuvre exaltante à l'échelle des maux qui frappent l'humanité, mais qui est aussi à la mesure des dévouements qu'elle suscite.

Georges Olivet avait trouvé dans sa famille, dans sa paroisse, dans sa ville, maintes occasions de dépenser son amour du prochain et voici que, dans ce vaste Congo, le Comité international de Genève lui permet de réaliser encore mieux le besoin qui est en lui de servir, de se dévouer et de s'accomplir.

Tous ceux qui ont été ses camarades et, tout de suite, ses amis, en témoignent par ma bouche. Grâce à lui, les délégués du Comité international ont pu remplir leur tâche en ayant à leurs côtés un conseiller et un réalisateur, à chaque instant disponible.

Toujours calme et souriant, exempt de cette nervosité qui atteint presque tous les blancs dans le climat débilitant de l'A frique centrale, Georges Olivet est prêt à toutes les besognes, même les plus humbles: il retient les places d'avion, procure tout ce qui est nécessaire à ses collègues, mais aussi, grâce à sa popularité, mènage des entretiens avecle chef d'Etat et ses ministres. Il sait ce qu'il faut dire et ce qu'il faut faire. Partout il est le bienvenu, et les portes qui s'ouvrent devant lui laissent passer à sa suite l'action bienfaisante de la Croix-Rouge.

Puis vient, cet automne, la mission dernière, la plus dure, celle pour laquelle il donnera toute sa mesure, l'intervention du Comité international au cours de l'action des Nations Unies au Katanga. Il s'agit, en plein combat, d'aller chercher des victimes qui gisent dans les rues, d'assurer l'hospitalisation des blessés, d'organiser l'évacuation des civils, de négocier des trêves indispensables, en un mot, de permettre à la croix rouge sur fond blanc, d'apporter, une fois de plus, sa protection charitable.

Cette lutte est aveugle et frappe au hasard. Dans des conditions qu'une enquête établira, je l'espère, Georges Olivet cherche en vain à 
franchir plusieurs fois les lignes. C'est lors d'une ultime tentative qu'il est frappé ainsi que ses deux compagnons.

Quoi qu'il en soit, le sacrifice est consommé, et le serviteur est tombé en pleine action, au moment oì son devoir remplissait son esprit et son cceur.

Lorsque, à la veille de Noël, je disais aux collaborateurs du Comité international l'angoisse que me causait le sort encore incertain de notre délégué au Katanga, j'ajoutais qu'en toutes circonstances, il y aurait pour nous une raison de croire et d'espérer puisque Georges Olivet nous avait affermi dans l'utilité ct la grandeur de notre tâche...

Après le jeu d'orgue, un pasteur célébra le culte funèbre, au cours duquel il évoqua la personnalité du défunt qui, en accomplissant sa tâche jusqu'au sacrifice suprême, illustra le verset de l'Evangile selon saint Jean: "Il n'est pas de plus grand amour que de donner sa vie pour son prochain ".

La mort brutale de Georges Olivet et les circonstances dans lesquelles elle s'est produite, le fait aussi que le signe de la croix rouge ne suffit plus aujourd'hui à protéger ceux qui, légitimement, s'en réclament, ont bouleversé la conscience universelle, comme il ressort des nombreux messages de condoléances qui ont affué à Genève, dès le 23 décembre I96I.

La Ligue de même que de nombreuses Sociétés nationales de la Croix-Rouge ont exprimé leur sympathie au CICR. En voici quelques témoignages ${ }^{1}$ :

Frappé par la triste nouvelle, transmets personnellement et au nom de la Croix-Rouge irlandaise profonde sympathie - Barry, Chairman.

La Croix-Rouge britannique profondément frappée par la mort tragique $\mathrm{M}$. Olivet, fait part de sa très profonde sympathie à la famille et au CICR - Limerick British Red Cross.

La Croix-Rouge de Belgique profondément émue s'associe au deuil qui frappe le Comité international de la Croix-Rouge, elle s'incline

${ }^{1}$ Certains d'entre eux sont traduits par nos soins. 
devant la mémoire de G. Olivet qui a mérité de la Croix-Rouge tout entière en donnant sa vie au service de l'humanité - Prince Albert de Belgique.

Apprends par presse mort Olivet et collaborateurs stop en vous priant transmettre vives condoléances familles éprouvées exprime admiration pour représentants tombés champ d'honneur avec sentiments horreur pour cette nouvelle atteinte privilèges Croix-Rouge - Baron Zeeland trésorier général Ligue des Sociétés de la Croix-Rouge.

Surpris apprendre nouvelle de presse et regrettons profondément décès tragique de Monsieur Georges Olivet délégué CICR en mission traditionnelle près Elisabethville stop Veuillez bien agréer notre condoléance sincère et transmettre nos sympathies cordiales à famille du défunt - Shimadzu président Croix-Rouge japonaise.

Douloureusement émue par mort Monsieur Olivet et ses compagnons tués au service de Croix-Rouge stop la Croix-Rouge française adresse au CICR expression sa sympathie profondément attristée — François Poncet, président Croix-Rouge française.

Emus apprendre sort tragique monsieur Georges Olivet et ses collaborateurs prions accepter nos plus profondes condoléances - Ferri président général Croix-Rouge italienne.

Nous sommes profondément affligés par la nouvelle du décès de $M$. Olivet, Nicole Vroonen et Styts Smeding, à la suite d'inexplicable accident et nous nous inclinons devant sacrifice d'un collaborateur de la CroixRouge qui au-delà de ce que lui commandait le devoir, a donné un grand exemple de dévouement à l'humanité - Philippine National Red Cross.

De nombreuses institutions ont également manifesté la part qu'elles prenaient à ce deuil. Entre autres, les Nations Unies, dont le secrétaire général, M. Thant, le fit dans les termes suivants:

J'ai l'honneur d'accuser la réception de votre message du 24 décembre relatif à l'annonce de la découverte des corps de Monsieur Olivet, Madame Vroonen et Monsieur Smeding stop je tiens à vous exprimer le profond chagrin que me cause le décès en service de ces hommes et de cette femme qui, conformément aux plus nobles traditions de la Croix-Rouge, se sont avancés au centre des combats pour accomplir leur mission humanitaire. Les représentants de l'Onuc ont eu de nombreuses occasions d'apprécier la qualité exceptionnelle du travail accompli par monsieur Olivet ainsi que sa contribution dans un esprit de dévouement total et de sacrifices personnels pour porter remède aux souffrances humaines pendant les hostilités récentes à Elisabethville. 
Indiquons enfin que le président du Katanga, M. Tschombé, de même que le ministre des Affaires étrangères du Congo, M. Bomboko, ont envoyé au CICR des télégrammes dont voici l'essentiel:

Gouvernement du Katanga vous présente sincères condoléance:; et tient à vous remercier de tout ce que Croix-Rouge a fait au Katanga depuis début des événements cruels que notre pays a connus, tient à vous assurer de sa plus complète collaboration avec une organisation qui ne cesse de s'attirer le respect de tous - Président du Katanga Moïse Tschombé.

Gouvernement et peuple congolais ont appris avec tristesse disparition délégué Comité international Croix-Rouge au Congo et de ses compagnons stop vous prie recevoir condoléances sincères gouvernement congolais et vous prie également présenter condoléances du gouvernement auprès de familles des défunts stop sens responsabilité et impartialité avec laquelle Monsieur Olivet a accompli sa mission au Congo ne peut que faire honneur au Comité international - Bomboko ministre Affaires étrangères.

On voit, par ces quelques citations déjà, l'émotion profonde qu'a causée la nouvelle de la mort du délégué du CICR, tué alors qu'il accomplissait le plus haut devoir que l'homme puisse se donner dans le monde, servir son prochain. Mais Georges Olivet ne s'est pas sacrifié en vain. Il est allé sans arme, dans une ambulance, et la guerre l'a tué, croyant tuer, avec lui et ses compagnons, l'esprit de paix et de bonne volonté qu'ils représentaient. Mais cet esprit ne peut être détruit : il demeure une des seules espérances aujourd'hui, dans un monde déchiré. 